\title{
Estimation of the Minimum Number of Tracks for SAR Tomography
}

\author{
Matteo Nannini, Rolf Scheiber, and Alberto Moreira, Fellow, IEEE
}

\begin{abstract}
Synthetic aperture radar tomography (SARTom) is the natural extension of SAR interferometry to solve for multiple phase centers within a resolution cell and obtain the 3-D representation of a scene. This paper deals with the determination of the minimum number of tracks required to perform SARTom. Through the prolate spheroidal wave functions, the number of equivalent targets of a volumetric source is derived, and from it, the minimum number of observations required to apply subspace superresolution methods is computed. The minimum tomographic aperture length is also investigated. The results are validated on real data acquired in L-band by the experimental SAR system of the German Aerospace Center.
\end{abstract}

Index Terms-Synthetic aperture radar tomography (SARTom), superresolution, system dimension.

\section{INTRODUCTION}

$\mathbf{S}$ YNTHETIC aperture radar tomography (SARTom) is an imaging technique that allows multiple phase center separation in the vertical (height) direction, leading to a 3-D reconstruction of the imaged scene. It is performed after standard 2-D SAR processing and operates on a stack of coregistered SAR images. Retrieval of volume structure information (e.g., for forest classification) and the solution of the layover problem are two of the most promising applications.

SAR interferometry (InSAR) [1], [2] is a first step toward 3-D imaging, but it only allows the recovery of information related to the mean phase center of all the targets present in a resolution cell. Polarimetric SAR interferometry (PolInSAR) [3] allows a phase center separation between targets within a resolution cell if their polarimetric behavior is different, but it fails to resolve them when they exhibit the same scattering mechanism. In this context, SARTom enhances the information extraction and allows one to obtain a complete 3-D representation of the scene. In [4], the first demonstration of airborne SARTom, using Fourier beamforming techniques, has been carried out, and the main constraints in terms of resolution and ambiguity rejection have been analyzed. The desired resolution specifies the length of the tomographic aperture $L_{\text {tomo }}$. On the other hand, the ambiguity height $V_{\mathrm{amb}}$ defines the baseline $d$ between the acquisitions (flight tracks). For the sake of simplicity,

Manuscript received February 11, 2008; revised April 30, 2008 and July 15,2008 . Current version published January 28,2009 . This work was supported in part by eOsphere Ltd. and by the Electro-Magnetic Remote Sensing Defence Technology Centre.

The authors are with the German Aerospace Center (DLR), Microwaves and Radar Institute, 82230 Oberpfaffenhofen, Germany.

Color versions of one or more of the figures in this paper are available online at http://ieeexplore.ieee.org.

Digital Object Identifier 10.1109/TGRS.2008.2007846 a linear regularly distributed acquisition geometry is assumed. The number of required tracks in order to obtain the desired performance is

$$
N=\frac{L_{\text {tomo }}}{d}+1
$$

Considering now the typical acquisition geometry of airborne systems (e.g., the Experimental SAR (E-SAR) system of the German Aerospace Center (DLR) [6]), it has been demonstrated [4] that for 2-3 m resolution in height a number of acquisitions ranging between $N=13$ and $N=20$ is required.

This large number of tracks required for SARTom makes it an expensive and, for a large volume thickness, an unfeasible task. A reduction on the number of passes is of fundamental importance in order to exploit tomography for future spaceborne missions. In [7] and [8], the potential of spaceborne SARTom has been experimentally demonstrated and the possibility to extend it to the 4-D case, based on the theory described in [9] (the so-called differential tomography) by adding the time dimension, has been shown and preliminary results have been presented in [10] and [11].

In recent years, it has been shown that extending direction of arrivals (DOAs) estimation techniques [12] to SARTom [13], [14], the Fourier resolution can be overcome, and therefore, it is supposed that the length of the synthetic aperture can be reduced without impacting the system capability to solve for targets in the height direction. This reduction will result in less flight tracks to be performed. In [4], the horizontal baseline $d$, required to avoid ambiguities, is defined as

$$
d \leq \frac{\lambda r_{0}}{2 V_{\mathrm{amb}}} \tan \left(\theta_{0}\right)
$$

where $\lambda$ is the wavelength, $V_{\mathrm{amb}}$ is the expected volume height, $r_{0}$ is the slant range distance, and $\theta_{0}$ is the look angle of the central track to the volume center.

In this paper, the minimum system dimension for SARTom is investigated for the case when subspace methods are applied to generate 3-D images. The conventional method to estimate the minimum number of tracks based on Fourier beamforming is not applicable in our case because of the different resolution that subspace methods can offer. More precisely, subspace methods can be applied if some minimum requirements in terms of signal and noise subspace are guaranteed. A useful mean to evaluate the expected dimension of such subspaces for band limited signals (as in the case of SARTom [4]) consists of the prolate spheroidal wave functions [22]. 


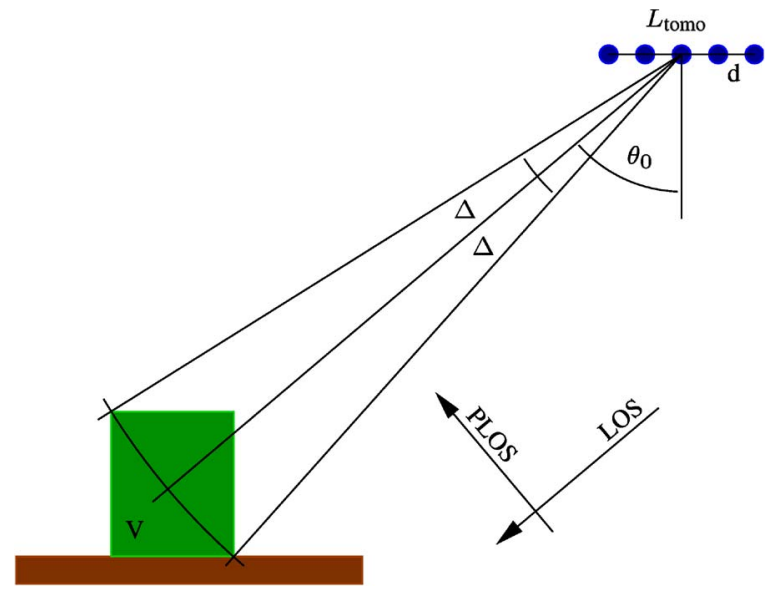

Fig. 1. Tomographic constellation and angular dispersion parameters. Lineof-sight (LOS) and perpendicular line-of-sight (PLOS) directions.

This paper is organized as follows. In the next section, the problem will be formulated and a brief explanation on the use of the MUSIC algorithm for SARTom will follow. In Section III, a model of a distributed source extended within the volume $V$ is described, and in Sections IV and V, the minimum number of tracks and the minimum tomographic aperture length for subspace methods are derived. In Section VI, numerical simulations will clarify the theoretical analysis, and in Section VII, the developed theory will be applied on experimental data acquired in L-band by the E-SAR system of the DLR. Section VIII summarizes this paper content.

\section{PRoblem Formulation}

The information content of a SAR resolution cell is the projection of the 3-D scattering contributions into a 2-D plane. The lost dimension is related to the height of the scatterers that are concurring to form the radar echo that will be compressed into the resolution cell. Assuming that the scattering mechanism verifies the Born approximation, ${ }^{1}$ after standard 2-D SAR processing [15], the information content of a resolution cell for the range-azimuth coordinate $(r, a)$ can be written as

$$
s(r, a)=\int_{\theta_{\min }}^{\theta_{\max }} \gamma(\theta) \exp \left(j \frac{4 \pi}{\lambda} r\right) d \theta
$$

where $\gamma(\theta)$ represents the complex reflectivity function of point scatterers located at a distance $r$ from the sensor in the direction $\theta$ from the track position (see Fig. 1). Obviously, there is a direct relation between the height of the scatterer, and the angle $\theta$ once $r$ is defined.

Considering now a finite number of scatterers $N_{\mathrm{s}}$ within the range of $\theta \in\left[\theta_{\min }, \theta_{\max }\right]$, it is possible, by means of a matrix

\footnotetext{
${ }^{1}$ The Born approximation represents the total scattered field as the superposition of the scattered fields generated by the single scatterers, neglecting the interactions between the scatterers themselves.
}

formulation, to represent the ensemble of the signals acquired by the tomographic constellation as

$$
\boldsymbol{x}=\boldsymbol{A \gamma}+\boldsymbol{n}
$$

where $\boldsymbol{x} \in \mathbb{C}^{N}$ represents the set of the $N$ focused signals for a $(r, a)$ position, $\boldsymbol{A} \in \mathbb{C}^{N \times N_{\mathrm{s}}}$ contains the so-called steering vectors $\boldsymbol{a}(\boldsymbol{\theta}) \in \mathbb{C}^{N}$

$$
\boldsymbol{a}(\boldsymbol{\theta})=\exp \left(j \frac{4 \pi}{\lambda} \boldsymbol{r}(\boldsymbol{\theta})\right)
$$

with $\boldsymbol{r}\left(\theta_{i}\right)=\left[r_{1}\left(\theta_{i}\right), \ldots, r_{N}\left(\theta_{i}\right)\right]$ denoting the distances between the scatterer located at $\theta=\theta_{i}$ and the array of SAR sensors; $\boldsymbol{n}$ represents an additive noise components.

There are several noise sources related to these kind of acquisitions. One of the most severe, particularly when vegetated areas are imaged with repeated passes, is provoked by the temporal decorrelation between the acquisitions themselves.

It is well known that in order to estimate the DOAs of the $N_{\mathrm{s}}$ scatterers, the MUSIC algorithm [16] represents one of the most important tools. The basic idea behind the algorithm is to define two subspaces: signal and noise subspace. After that, operating on the steering vector, it is possible to scan a functional that represents the projection of the steering vectors themselves into the noise subspace. If the steering vector is matching a direction where a scatterer is located, then its projection into the noise subspace will be zero. The functional of the MUSIC algorithm is defined by the inverse of this projection

$$
P_{\mathrm{MU}}\left(\theta_{I}\right)=\frac{1}{\boldsymbol{a}^{H}\left(\theta_{i}\right) \boldsymbol{E}_{\boldsymbol{N}} \boldsymbol{E}_{N}^{H} \boldsymbol{a}\left(\theta_{i}\right)}
$$

where $H$ stands for conjugate transpose and $\boldsymbol{E}_{\boldsymbol{N}}$ is an $N \times\left(N-N_{\mathrm{s}}\right)$ matrix whose columns are the eigenvectors of the sample covariance matrix that span the noise subspace.

Since in reality, point-like responses are not common and, particularly for SARTom, the great interest is given by volumetric structures; in the next section, a distributed source model is introduced to define an equivalent number of sources that could be employed to define the dimension of an effective signal space [18], [19] and, consequently, to derive the expression of the number of tracks required to image this kind of sources.

The MUSIC algorithm has been successfully used for SARTom applications [14]. The results over forested areas agree with the analysis [20] in the field of sonar applications that involve distributed sources. In particular, the application of the MUSIC algorithm allows a first estimation of the mean phase center of the volumetric target and its width. The MUSIC algorithm is often defined as a pseudobeamforming technique because, for radar applications, it gives information related to the position of the phase centers of the scatterers, rather than a measure of their backscattered power. For this reason, once the number of phase centers $N_{\mathrm{S}}$ is defined, only the first $N_{\mathrm{s}}$ peaks of the response have relevance. In order to design a tomographic constellation, when such subspace methods are used, it is of fundamental importance to evaluate the expected behavior of the eigenvalues of the covariance matrix as we shall see in the next sections. 


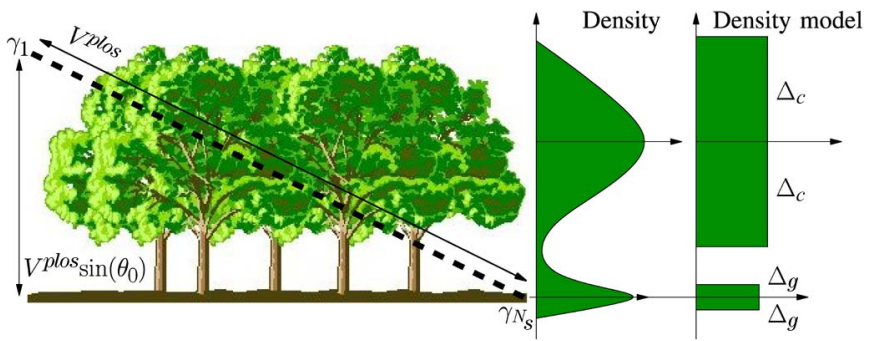

Fig. 2. Distributed scatterer model: Gaussian distribution and uniform density model. $\Delta_{\mathrm{C}}$ and $\Delta_{\mathrm{g}}$ are the half of the angular dispersions of the canopy and the ground, respectively. The quantity $V^{\text {plos }}$ is depicted as well as a discrete distribution of $N_{\mathrm{S}}$ scatterers for the considered slant-range coordinate.

\section{Distributed Source Model}

In order to assign a dimension to the signal subspace, the number of sources has to be defined. This parameter can be estimated from the rank of the sample covariance matrix

$$
\boldsymbol{R}=\frac{1}{K} \sum_{k=1}^{K} \boldsymbol{x}(k) \boldsymbol{x}^{H}(k)
$$

where $K$ is the number of the snapshots. In the case of distributed scatterers, this matrix will have full rank, therefore the noise subspace will have dimension equal to zero and MUSIClike algorithms cannot be used [18]. Despite that, the greater part of the energy is often concentrated in few eigenvalues. The identification of this number of dominant eigenvalues will represent the effective signal space dimension [18], [19] that will be called $N_{\text {efs }}$.

In this section a scatterer model will be defined to allow the estimation (in Section IV) of $N_{\text {efs }}$ of this scenario by means of the prolate spheroidal wave functions. Once this parameter is estimated, it will be possible to define the minimum number of required observations (tracks) in order to guarantee that the effective noise subspace will have its dimension greater than zero.

One worst case scenario for SARTom consists of a volumetric scatterer like a forest because of its large number of scatterers. If a SARTom system could guarantee a 3-D imaging of these structures, then for other types of environment, where the number of scatterers is lower, the performance of the system does not drop down (cf., simulations in [18]). For this reason, it will be assumed a forest-like distributed target as a reference one.

In order to do that, the model introduced in [17] will be considered. The signal attenuation, proportional to the density of the scatterers, is modeled as the sum of a crown contribution with a Gaussian shape and the ground contribution modeled as $\delta(\cdot)$ function. In [5], it has been experimentally proved the validity of such a model and the possibility for SARTom to recover the density information. In reality, also the ground contribution will present a Gaussian shape (with smaller standard deviation compared to the canopy), therefore both sources will be modeled as Gaussian distributed sources (Fig. 2). Since ground and canopy do not overlap, no superposition between the sources will be assumed. The sum of their angular dispersion is limited by $2 \Delta$.
In [18], it has been shown that, if a uniform distribution is assumed, the widest spread of the signal energy among the dominant eigenvalues is achieved. This implies that a uniform distribution represents the worst case scenario. For this reason, two uniformly distributed scatterers with their main phase centers located on the density peak of the canopy and on the ground, respectively, will be assumed (see Fig. 2). The distribution of a uniformly distributed scatterer will be

$$
P_{i}(\theta)=\alpha_{i} \begin{cases}\frac{1}{2 \Delta_{i}} & \text { if }\left|\theta-\theta_{i}\right| \leq \Delta_{i} \\ 0 & \text { otherwise }\end{cases}
$$

where $i=1,2$ (canopy, ground) and $\theta_{i}$ indicates the angle related to the position of the mean phase center of the $i$ th scatterer, $\Delta_{i}$ corresponds to half of its extension in radians, and $\alpha_{i}$ is a constant term.

\section{Minimum Number OF TRACKS}

Following [18] and [19], it is possible to analyze the effective dimension of the signal space of the volumetric target defined in the previous section. Due to the Born approximation, the two distributed scatterers are assumed to be uncorrelated to each other, and therefore, the sample covariance matrix can be written as

$$
\boldsymbol{R}=\sum_{i=1}^{2} \boldsymbol{R}_{i}=\boldsymbol{R}_{1}+\boldsymbol{R}_{2}
$$

where $\boldsymbol{R}_{1,2}$ are the sample covariance matrices related to the canopy and the ground, respectively. The effective rank of $\boldsymbol{R}$ gives the number of effective sources $N_{\text {efs }}$, and it is upper bounded by

$$
\operatorname{rank}(\boldsymbol{R}) \leq \operatorname{rank}\left(\boldsymbol{R}_{1}\right)+\operatorname{rank}\left(\boldsymbol{R}_{2}\right)
$$

where $\operatorname{rank}(\cdot)$ means effective rank.

At this point, this paper will be focused on the estimation of the number of effective sources of a single uniformly distributed scatterer. When this task will be solved, one would only need to perform the analysis for the canopy and the ground component separately, and then, the global $N_{\text {efs }}$ will be given by the sum of the two estimated dimensions. In fact, we shall see (Appendix A) that the worst case scenario will converge to a single uniformly distributed scatterer of height $V$.

For the following analysis, a sufficient signal to noise ratio is assumed. In term of an eigenvalue analysis (introduced in the next section), the effect of $\boldsymbol{n}$ will mainly impact the power of weak eigenvalues (that will be comparable to the noise one), increasing the noise subspace.

\section{A. Number of Dominant Eigenvalues Estimation}

To determine the number of dominant eigenvalues of a distributed source, a continuous acquisition along the tomographic aperture is first assumed. The concept will be then generalized to a discrete acquisition geometry in Section IV-B. 
For a single contribution, the form of the generic cross correlation in a continuous acquisition case and under the model assumptions of Section III is

$$
\begin{aligned}
E\left[x(l) x^{*}\left(l^{\prime}\right)\right] & =\int_{-\frac{\pi}{2}}^{+\frac{\pi}{2}} \exp \left(j \frac{4 \pi\left(r_{l}(\phi)-r_{l^{\prime}}(\phi)\right)}{\lambda}\right) P(\phi) d \phi \\
& =\int_{\theta_{0}+\Delta}^{\theta_{0}-\Delta} \exp \left(j \frac{4 \pi\left(l-l^{\prime}\right) \sin (\phi)}{\lambda}\right) \frac{1}{2 \Delta} d \phi
\end{aligned}
$$

where $l-l^{\prime}$ is the distance between the sensors located at the coordinate $l$ and $l^{\prime}$. Now, substituting $\phi^{\prime}=\phi-\theta_{0}$ with $\phi^{\prime} \in$ $[-\Delta,+\Delta]$ and assuming $\Delta$ small (true for typical SAR acquisition geometry), $\sin \left(\phi^{\prime}+\theta_{0}\right) \simeq \sin \left(\theta_{0}\right)+\phi^{\prime} \cos \left(\theta_{0}\right)$ and the following equality holds:

$$
\begin{aligned}
E\left[x(l) x^{*}\left(l^{\prime}\right)\right]=\exp ( & \left.j \frac{4 \pi}{\lambda}\left(l-l^{\prime}\right) \sin \left(\theta_{0}\right)\right) \\
& \times \operatorname{sinc}\left(\frac{4}{\lambda}\left(l-l^{\prime}\right) \cos \left(\theta_{0}\right) \Delta\right) .
\end{aligned}
$$

At this point, the dimension of the effective signal subspace can be estimated through an eigenvalues analysis of (12)

$$
\begin{aligned}
& \int_{-\frac{L_{\text {tomo }}}{2}}^{+\frac{L_{\text {tomo }}}{2}} \exp \left(j \frac{4 \pi}{\lambda}\left(l-l^{\prime}\right) \sin \left(\theta_{0}\right)\right) \\
& \quad \cdot \operatorname{sinc}\left(\frac{4}{\lambda}\left(l-l^{\prime}\right) \cos \left(\theta_{0}\right) \Delta\right) \Phi_{n}\left(l^{\prime}\right) d l^{\prime}=\mu_{n} \Phi_{n}(l) .
\end{aligned}
$$

In [4], the band limited nature of the tomographic signal has been demonstrated. Under this assumption, the eigenproblem (13) can be faced by means of the prolate spheroidal wave functions [22]. Indeed, the eigenvalues $\mu_{n}$ will have the form

$$
\mu_{n}=\frac{2 c}{\pi}\left[R_{0 n}^{(1)}(c, 1)\right]^{2}
$$

where $R_{0 n}^{(1)}(c, 1)$ are the radial prolate spheroidal functions and the expression of the parameter $c$, the so-called spacebandwidth product, is given by (for further details see Appendix B)

$$
c=\frac{2 \pi \Delta \cos \left(\theta_{0}\right)}{\lambda} L_{\text {tomo }} .
$$

The most of the energy is concentrated in the first $\lceil 2 c / \pi\rceil$ eigenvalues [22] ( $\lceil\cdot\rceil$ represents the next integer greater than the argument). Therefore, it can be concluded that for the SARTom case, the number of effective scatterers is

$$
N_{\mathrm{efs}}=\left\lceil\frac{2 c}{\pi}\right\rceil=\left\lceil\frac{4 \Delta \cos \left(\theta_{0}\right)}{\lambda} L_{\mathrm{tomo}}\right\rceil .
$$

It is possible to observe that there is a linear relation between the tomographic aperture and the parameter $N_{\text {efs }}$, because the larger the aperture, the more uncorrelated will be the signal

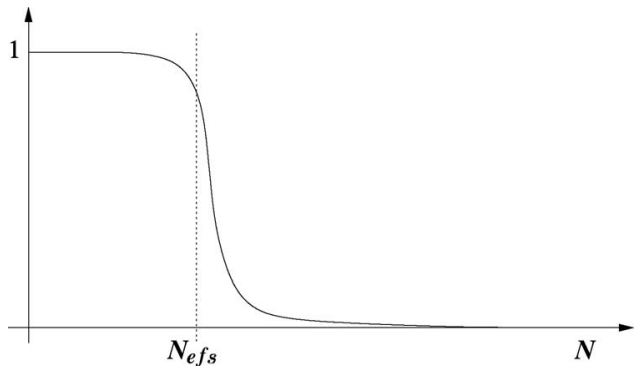

Fig. 3. Typical behavior of the magnitude of the eigenvalues (normalized).

sampled by the different acquisition tracks. In Section VI, a practical description of this characteristic will be presented.

\section{B. Noncontinuous Acquisition Case: SARTom}

In the previous section, an analysis related to a continuous acquisition geometry of sensors distributed along the tomographic aperture has been carried out. Now, in order to extend such an analysis to the SARTom case, a discrete acquisition geometry has to be assumed. A continuous acquisition can be seen, at the limit, as the one that would result from an infinite sampling frequency along the tomographic aperture. Indeed, this oversampling along the tomographic aperture axis, allows one to describe the decay of the eigenvalues through the prolate spheroidal wave functions (Fig. 3).

Starting from a continuous acquisition, a decrease of the system dimension ( $d$ increases) will not affect the shape of the behavior presented by the eigenvalues because, due to the fact that only eigenvalues with negligible energy are discarded, the amount of information does not change. This is true until $N-1$ is approaching $N_{\text {efs. }}$. In this case, we have

$$
\frac{L_{\text {tomo }}}{d}=\frac{4 \Delta \cos \left(\theta_{0}\right)}{\lambda} L_{\text {tomo }} .
$$

Therefore

$$
d=\frac{\lambda}{4 \Delta \cos \left(\theta_{0}\right)} .
$$

Now, defining $V^{\text {plos }}$ as the volume height in the direction perpendicular to the line-of-sight (PLOS), it results that

$$
\begin{aligned}
\Delta & =\frac{V^{\text {plos }}}{2 r_{0}} \\
\sin \left(\theta_{0}\right) & =\frac{V}{V^{\text {plos }}} .
\end{aligned}
$$

With simple derivation, it is possible to demonstrate that (18) is equal to (2). In Fig. 3, the typical behavior of the eigenvalues is depicted, and $N_{\text {efs }}$ is represented.

This result is consistent to the fact that the $N_{\text {efs }}$ is known as the Shannon number and represents the number of sampling points spaced by the Nyquist distance $d$ [21]. A direct consequence of (17) is that $N \geq N_{\text {efs }}+1$. If $N$ is large enough to represent the first weak eigenvalue that is the goal of our analysis in order to guarantee the existence of the smallest effective noise subspace, the continuous analysis can be extended to the discrete case. Therefore, an oversampling along the tomographic aperture is required. We shall see in the next 
section that this drawback is compensated by a strong reduction of the minimum required tomographic aperture length that subspace methods require.

In addition, the continuous case will allow the closest estimation of the actual $N_{\text {efs }}$ for the chosen value of $L_{\text {tomo }}$ (due to the oversampling) that is one of the most important parameters to consider if going for a system reduction.

In order to allow the system to describe the viewed volume, no underestimation of the effective signal subspace is allowed. Indeed, no dominant eigenvalues have to be excluded. Therefore, the maximum distance between tracks $d_{\mathrm{Nyq}}$ can be exploited in order to obtain a starting candidate for the minimum number of tracks. As explained, spacing the tracks with $d=d_{\mathrm{Nyq}}$ implies that only the eigenvalues that have magnitude close to one can be viewed; therefore, it will be necessary to reduce $d$ to represent also eigenvalues with important magnitude and the effective noise subspace. Hence, it has to be guaranteed that through the eigenvalues decomposition the following conditions for $N$ holds:

$$
\begin{aligned}
& N=N_{\mathrm{efs}}+M \\
& \min _{N} \frac{\mu_{N-1}}{\mu_{0}} \simeq 0
\end{aligned}
$$

where $M$ is an integer value greater than one, and $\mu_{0}$ and $\mu_{N-1}$ are the strongest and the weakest eigenvalues, respectively. The tables in [23] will allow one to identify these parameters. Condition (22) means that we are considering the smallest system dimension because only one eigenvalue will be related to the noise subspace. It is understood that large temporal decorrelation between the acquisitions will impact the eigenvalues' behavior and reduce the information content of the data. For this reason, SARTom cannot be applied when the temporal baseline is large and the imaged scene is changing significantly between the acquisitions.

Until now, the length of the tomographic aperture has been assumed as known. In the next section, boundary conditions in order to define it are described.

\section{Minimum Tomographic Aperture Length}

First of all, it is necessary to describe how the effective signal subspace dimension related to the two main contributions of the canopy and the ground can be estimated. In Appendix A, it is shown how the analysis carried out for a single uniformly distributed source is still a worst case scenario if the two distributed sources do not overlap and are within the volume height $V$ (model assumptions). Therefore, a single uniformly distributed scatterer will be assumed. Despite the fact that for SARTom the overlap hypothesis is not realistic, in Appendix C derivations related to this case are reported for completeness.

The tomographic aperture dimension is given by (1), where the Nyquist criterion is playing an important role in the choice of the baseline. In order to determine the minimum number of tracks required to represent the volume, the smallest value of $L_{\text {tomo }}$ and, consequently of $c$ [see (15)], has to be identified.

Since the minimum number of dominant eigenvalues that one wants to retrieve is $N_{\text {efs }}=2$ (ground, canopy components

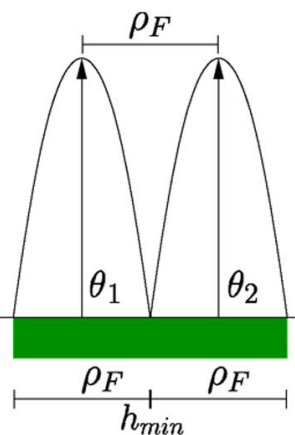

Fig. 4. Definition of the minimum angular separation of two sources located at $\theta_{1}$ and $\theta_{2}$. Comparison with standard Fourier beamforming: $\rho_{\mathrm{F}}$ is the Fourier geometric vertical resolution and $h_{\min }$ represents the minimum vertical volume height within the two sources can be solved.

from the model assumptions of Section III), this implies from (16) that

$$
c>\frac{\pi}{2} .
$$

Referring now to (15), it is possible to write the expression of $L_{\text {tomo }}$ as

$$
L_{\text {tomo }}>\frac{\lambda}{4 \Delta_{\min } \cos \left(\theta_{0}\right)}
$$

where $\Delta_{\min }$ is the half of the minimum angular dispersion (where the two sources have to be located) and has the expression

$$
\Delta_{\min }=\frac{h_{\min }}{r_{0} 2 \sin \left(\theta_{0}\right)}
$$

where $h_{\min }$ is the minimum height where the two sources are located. It is interesting to observe that with simple substitutions the minimum length of the tomographic aperture becomes

$$
L_{\text {tomo }}>\frac{\lambda r_{0}}{2 h_{\min }} \tan \left(\theta_{0}\right)
$$

that together with (18) constitutes the link of this paper to [4] with the exception that $h_{\min }$ takes the place of the Fourier tomographic resolution $\rho_{\mathrm{F}}$. It is important to remark that for subspace methods, it is not possible to find a direct expression for the resolution, because it depends on the data itself. At the same time, it is useful to find a link between the Fourier resolution and the minimum height $h_{\min }$ in order to evaluate the impact of the system reduction. Without loss of generality, it can be said that within a volume height of $2 \rho_{\mathrm{F}}$ two main contributions whose distance corresponds to $\rho_{\mathrm{F}}$ have to be identified (see Fig. 4). Therefore, once a reference resolution has been defined, the correspondent $h_{\min }$ and the minimum length $L_{\text {tomo }}$ can be identified.

\section{NUMERICAL EXAMPLES}

In order to clarify the previous analysis, three examples involving airborne and spaceborne systems operating with different frequencies will follow. 


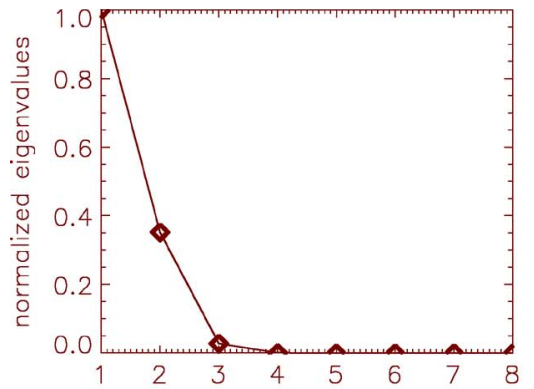

(a)

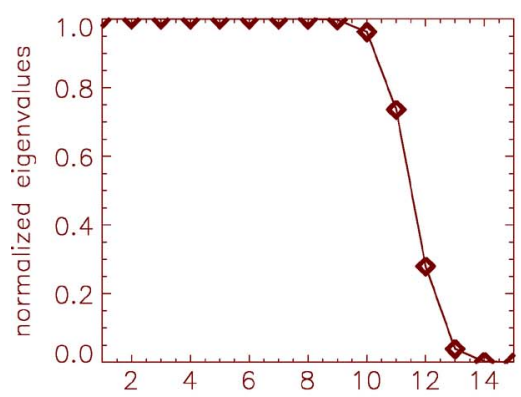

(c)

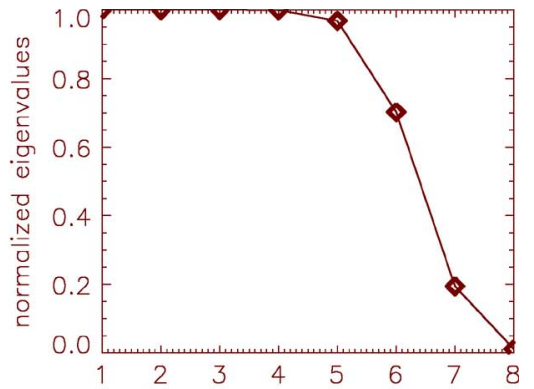

(b)

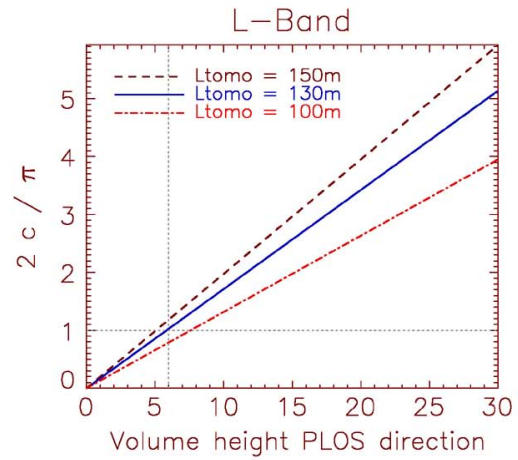

(d)

Fig. 5. (a) Eigenvalue diagram related to the minimum volume within which two distributed sources that have a distance of $3 \mathrm{~m}$ in the PLOS direction have to be represented. The minimum tomographic aperture length that results is $L_{\mathrm{tomo}}=130 \mathrm{~m}$. (b) Eigenvalue diagram correspondent to the maximum volume height viewed by the same tomographic constellation as in (a). (c) Effect of increasing the tomographic aperture from 130 to $250 \mathrm{~m}$ without changing the required baseline. (d) Value of $2 c / \pi$ (related to the number of dominant eigenvalues) as a function of the PLOS height and different values of the tomographic aperture $L_{\text {tomo. }}$. The minimum tomographic aperture length of $L_{\text {tomo }}=130 \mathrm{~m}$ is represented (solid line).

\section{A. Airborne Case: L-Band}

The scene to be viewed consists of a volume of a maximum height in the PLOS direction of $30 \mathrm{~m}$. The master track of the tomographic constellation is located in the center of it, and its height above ground is $H=3200 \mathrm{~m}$, the slant range coordinate related to the center of the scene is $r_{0}=4500 \mathrm{~m}$ (refer to Fig. 1). The frequency used is L-band corresponding to a wavelength $\lambda=24 \mathrm{~cm}$. With this geometry, the minimum allowed baseline is around $d_{\mathrm{Nyq}}=25 \mathrm{~m}$ (2).

Now, the minimum length of the tomographic aperture has to be determined. Proceeding as in Section V, defining an equivalent Fourier resolution of $\rho=3 \mathrm{~m}$ in the PLOS direction (and $\rho_{\mathrm{F}}=\rho \sin \left(\theta_{0}\right)$ in the vertical direction), the minimum volume height corresponds to $h_{\min }=2 \rho \sin \left(\theta_{0}\right)=4.3 \mathrm{~m}$. The minimum value of the tomographic aperture is $L_{\text {tomo }}=130 \mathrm{~m}$. This aperture will allow one to view the two components when their distance is $3 \mathrm{~m}$ in PLOS. The system dimension at this point is determined with the help of the maximum volume height, which is related to the maximum number of effective sources that the system will need to represent. From $d_{\mathrm{Nyq}}$, it is already possible to say that the number of acquisitions has to be greater than six, but precise boundary conditions are obtained computing the value of $c$ (15) for the maximum PLOS volume height of $30 \mathrm{~m}$ that is $c=8$. Now, referring to the tables [23] and to the conditions (21) and (22), a minimum number of acquisition of $N=8$ results. Therefore, the required baseline will be approximately $d=18 \mathrm{~m}$.

Comparing now this geometry with the one obtained by means of Fourier-based techniques, we obtain for the later one, a minimum tomographic aperture of $250 \mathrm{~m}$ with at minimum $N=11$ tracks, because of $d_{\mathrm{Nyq}}$. It is worth to mention that, due to the limited resolution inherent to the Fourier beamformer when compared with subspace methods, a few tracks are added for reducing the baseline value in order to ensure stable ambiguity rejection. In fact, in [4], $N=13$ passes have been flown in order to allow the system to perform as in this example.

Now that the constellation has been defined, an analysis of eigenvalue diagrams generated from (12) is carried out. Fig. 5(a) shows the eigenvalue diagram that results for the assumed $h_{\min }$. It is possible to observe that most of the energy is concentrated in the first two eigenvalues, as expected. Thus, the dimension of $L_{\text {tomo }}$ is sufficient to identify the two main contributions. Fig. 5(b) shows the situation when the maximum volume (of $30 \mathrm{~m}$ in the PLOS direction) is viewed by the tomographic constellation. The increase of the dominant components is a consequence of the fact that when a volume with the maximum height is viewed, the number of effective sources increases. In this particular case, it is possible to observe that from two main sources, six dominant eigenvalues represent the effective signal space [see (16)]. It is important to observe that for the minimum volume the main objective is to extract two main contributions, on the other hand, for the maximum volume, the minimum number of tracks has to allow the recovery of an effective noise subspace of dimension one. In other words, for this last case, all the eigenvalues with negligible magnitude, except one, can be discarded.

Referring to these geometry parameters, it is now possible to show how the number of dominant eigenvalues depends on the length of the tomographic aperture (cf., Section IV-A). For 


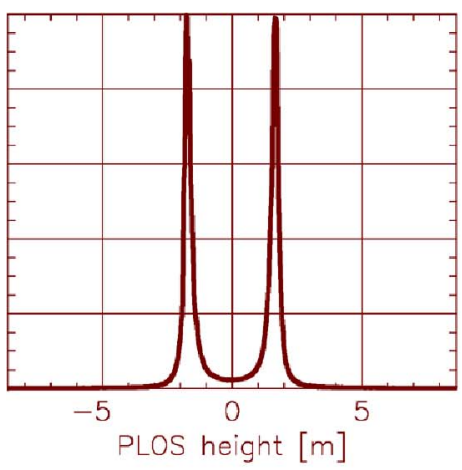

(a)

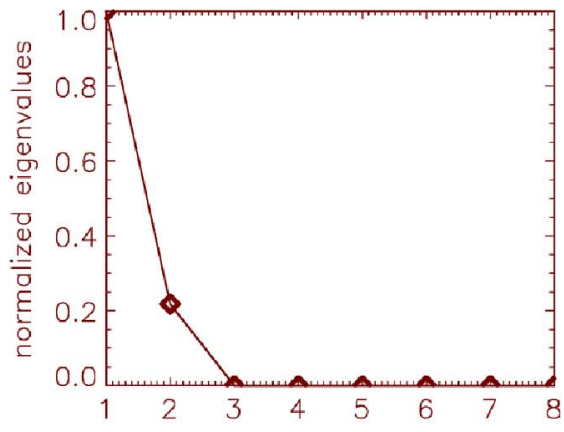

(b)

Fig. 6. Minimum angular separation simulation. The results are obtained by means of the MUSIC algorithm for the reduced acquisition geometry case. (a) Processing result and (b) eigenvalue diagram.

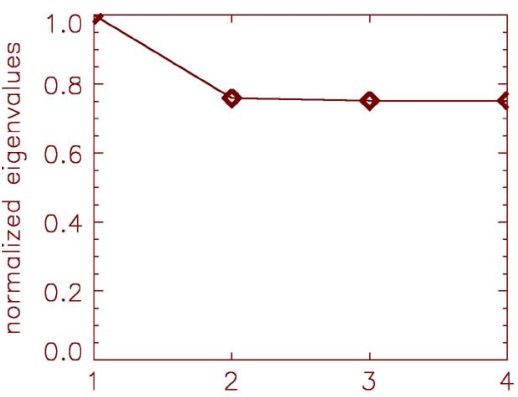

(a)

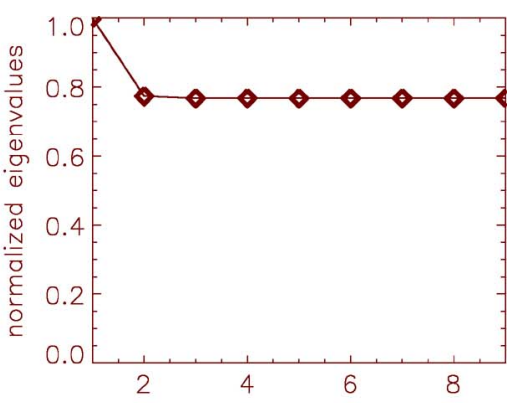

(b)

Fig. 7. Eigenvalue behavior when the Nyquist boundary condition is not respected. (a) $L_{\text {tomo }}=99 \mathrm{~m}$ with $d=33 \mathrm{~m}$ and (b) $L_{\text {tomo }}=208 \mathrm{~m}$ and $d=26 \mathrm{~m}$.

this reason, the maximum volume has been viewed by a constellation of $N=15$ tracks, spaced by a baseline $d=18 \mathrm{~m}$ obtained by previous analysis. Fig. 5(c) shows the correspondent eigenvalue diagram. Comparing Fig. 5(b) with Fig. 5(c), one can observe that despite of no change in the structure has been introduced, the increase of the tomographic aperture represents the same structure with a larger number of effective sources than before.

In Fig. 5(d), the value of $2 c / \pi$ [from which the value of $N_{\text {efs }}$ can be computed (16)], for different tomographic aperture behavior is represented as a function of the volume height. It is important to observe that the chosen aperture corresponds to the minimum aperture that can represent two dominant eigenvalues which are within a PLOS volume height of $6 \mathrm{~m}$. As the PLOS volume height is greater than $6 \mathrm{~m}$, the second eigenvalue starts to have nonnegligible energy.

In order to show that with this constellation, two contributions within $2 \rho$ can be separated, a simulation has been carried out. The signal related to several point sources (more than the number of tracks) located within a PLOS volume height of $6 \mathrm{~m}$ and acquired by the reduced constellation have been processed by means of the MUSIC algorithm. The density of the sources increases around two peaks that have a height difference of $3 \mathrm{~m}$ in PLOS. The sample covariance matrix has been built with 1000 snapshots. The result is shown in Fig. 6(a). It is possible to note that the locations of the phase centers are correctly recovered despite of the system reduction. Fig. 6(b) shows the relative eigenvalue diagram. As it is possible to see, most of the energy is concentrated in the first two eigenvalues, despite the higher number of scatterers, as expected from the theoretical analysis.

Another aspect that is worth to describe is related to the eigenvalues behavior when the maximum distance between tracks given by (18) is not respected. In Fig. 7, the eigenvalues behavior is reported for the tomographic aperture dimensions of $L_{\text {tomo }}=99 \mathrm{~m}$ and $208 \mathrm{~m}$ acquired with a baseline of $d=33$ and $26 \mathrm{~m}$, respectively. The maximum Nyquist distance is $d_{\min }=25 \mathrm{~m}$. The impact of aliasing in the acquisition of the data corresponds to eigenvalue diagrams that do not converge to zero.

It is understood that after the geometry has been determined, a feasibility analysis related to the baseline dimension and the tomographic aperture length has to be carried out in order to match the SAR processing requirements. In particular, the impact of temporal decorrelation has to be considered during the planning of the campaign in order to allow SARTom to be applied. However, the MUSIC algorithm will perform better than the conventional Fourier beamforming due to its capability to separe the noise from the information.

\section{B. Airborne Case: P-Band}

For the case of a P-band system, operating with a wavelength of $69 \mathrm{~cm}$ that is viewing the same scene described in the previous section with the same constraints in terms of minimum volume height, a tomographic aperture of $L_{\text {tomo }}=364 \mathrm{~m}$ and 


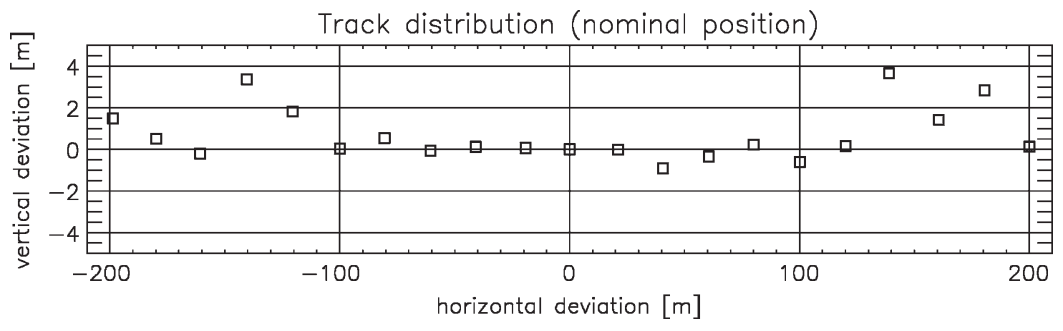

Fig. 8. Acquisition geometry: nominal tracks. $N=21$ tracks uniformly distributed with an average baseline of $20 \mathrm{~m}$ along a horizontal tomographic aperture of $400 \mathrm{~m}$.

$N=8$ tracks spaced by $d=52 \mathrm{~m}$ results. It is interesting to observe that, despite the wavelength is changed, the minimum number of tracks remains the same. This is a consequence of the fact that the scene and the target separation requirements did not change. Indeed, combining (15) with (24), one could see that the value of $c$ can be written as

$$
c>\frac{\pi}{2} \frac{\Delta_{\max }}{\Delta_{\min }}
$$

where $\Delta_{\max }$ corresponds to the maximum volume height. This equation shows how the minimum value of the parameter $c$ can be defined from the scene requirements and, once it is determined, the minimum number of tracks remains constant independently on the parameters (frequency, geometry) of the observation.

\section{Spaceborne Case: C-Band}

For the spaceborne case, a constellation with a master track located at a height above ground of $800 \mathrm{~km}$, an averaged look angle of $23^{\circ}$, and a wavelength of $5.6 \mathrm{~cm}$ have been considered (ERS-1 typical parameters). The minimum PLOS volume height requirement has been relaxed to $12 \mathrm{~m}$. It results a constellation of $N=5$ passes separated by a baseline of $d=550 \mathrm{~m}$ that covers an aperture of around $L_{\mathrm{tomo}}=2200 \mathrm{~m}$.

\section{EXPERIMENTAL RESULTS}

The theory described in the previous section is validated on real data. The E-SAR data set has been acquired in L-band during one day in September 2006 over Dornstetten (Germany) for target detection purposes [24]. The area where the experiment took place is relatively flat and half of the region is covered by nonhomogeneous forest stands related to different species. In average the tree height ranges between 10-25 m.

The acquisition geometry is nominally a regular horizontal grid of 21 tracks with an average baseline of $20 \mathrm{~m}$, resulting in a tomographic horizontal aperture of $400 \mathrm{~m}$. It is possible to observe (Fig. 8) that the actual acquisition geometry is very close to the planned one, with a maximum deviation of around $4 \mathrm{~m}$ between nominal and planned track. In order to perform tomography, the effects of the motion deviations typical of this kind of platform have to be compensated adaptively for each height [25].

The mid-range coordinate and the value of the look angle related to the center of the scene are practically identical to

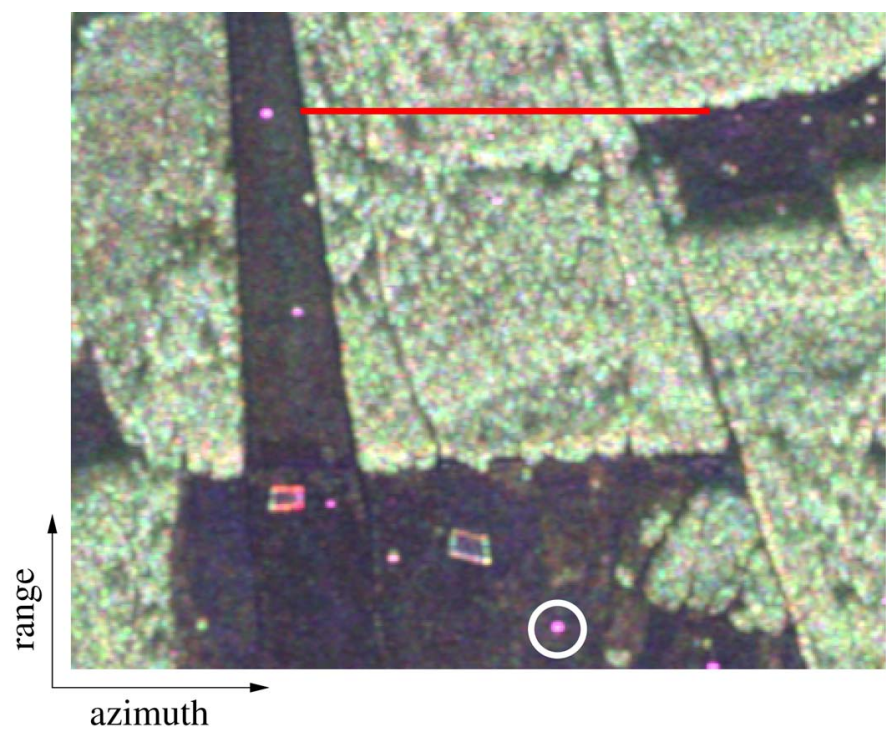

Fig. 9. Full polarimetric SAR composite master image with $\mathrm{R}(\mathrm{HH})-\mathrm{G}(\mathrm{HV})-$ $\mathrm{B}(\mathrm{VV})$ color coding. The forested area is analyzed along the cut represented by the straight line. The point identified by the circle represents two corner reflectors in a layover geometry.

the assumed scenario in the L-band airborne numerical example $\left(r_{0}=4500 \mathrm{~m}\right.$ and $\left.\theta_{0}=45^{\circ}\right)$.

In Fig. 9, the full polarimetric composite master image is shown. For the following analysis, the $\mathrm{HH}$ polarization will be considered. In order to maintain the requirements of the numerical example (minimum PLOS volume height of $6 \mathrm{~m}$ for the mid-range), the minimum suitable aperture length is $L_{\text {tomo }}=140 \mathrm{~m}$ acquired with $N=8$ passes. The line depicted over the forested area in Fig. 9 shows the cut along which the tomographic processing is performed. The considered cut is at a distance of $4816 \mathrm{~m}$ from the sensor that corresponds to a look angle of approximately $\theta_{0}=50^{\circ}$.

As shown, the behavior of the eigenvalues depends on the volume height [cf., (16)]. For a fixed value of $N$, the maximum allowable volume height corresponds to an eigenvalue diagram as the one of Fig. 5(b), where $N-1$ eigenvalues have nonnegligible magnitude, and the $N$ th one has magnitude close to zero to allow the definition of the smallest noise subspace. These conditions permit to identify the spacebandwidth product as $c=8$ from [23]. Before proceeding with the tomographic results, it is worth to check if the forest height along the chosen profile is less than the maximum allowable one. The boundary height can be computed combining (15), 


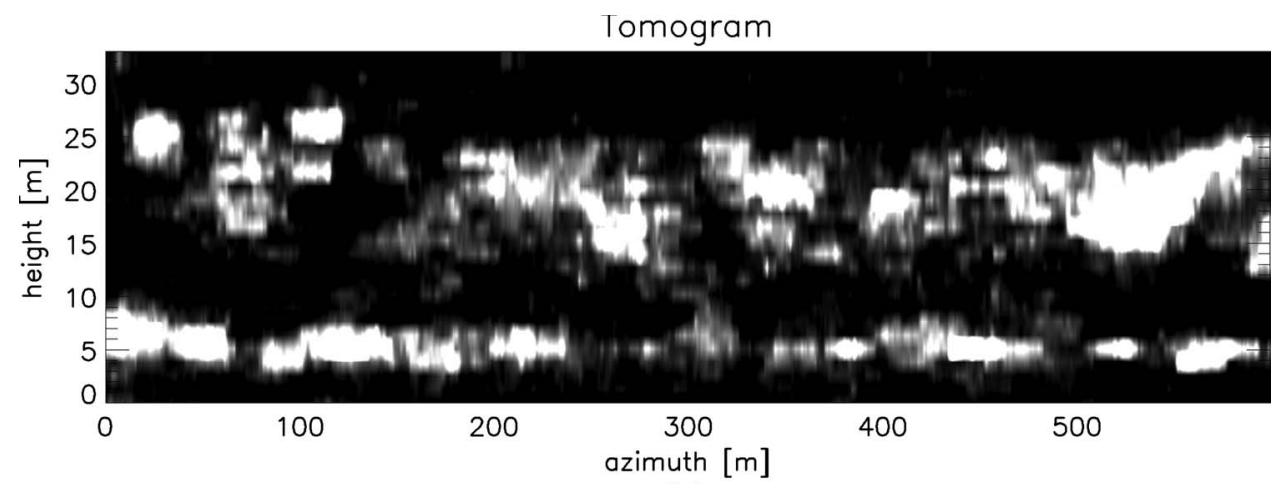

(a)

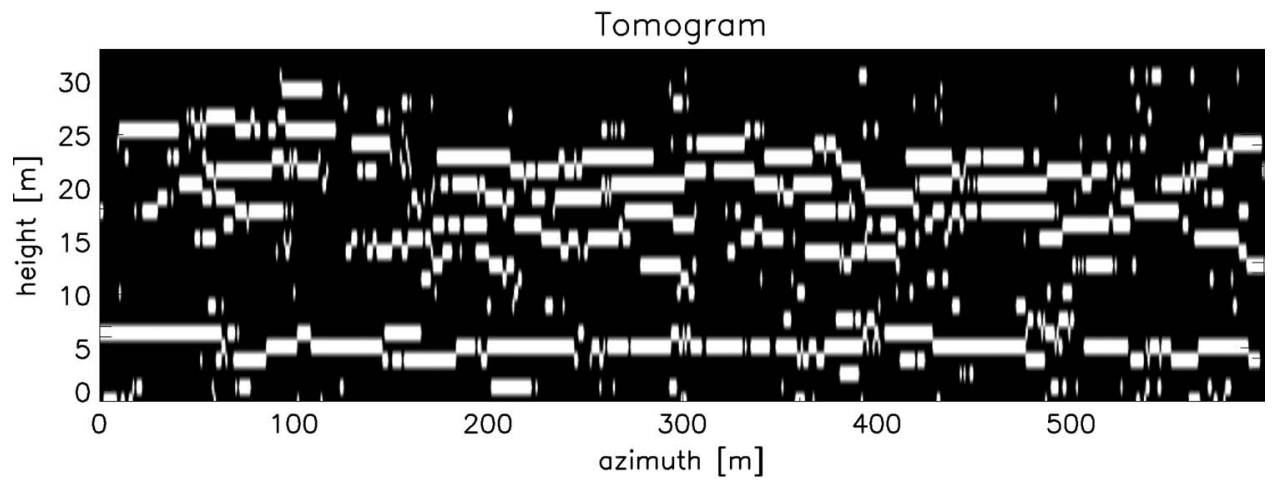

(b)

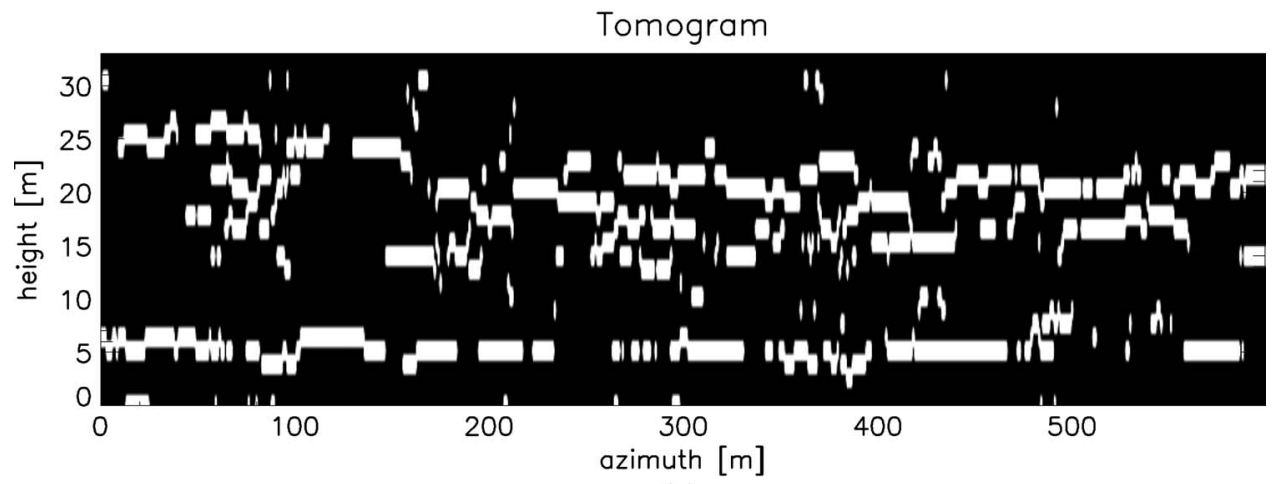

(c)

Fig. 10. Tomograms of a forested area obtained using the full tomographic aperture and $N=21$ tracks with (a) Capon beamforming and (b) MUSIC algorithm. (c) Tomogram obtained using the reduced aperture and $N=8$ tracks by means of the MUSIC algorithm.

(19), and (20). It results that, if the forest height along the cut is less than

$$
V=\frac{\lambda c \tan \left(\theta_{0}\right) r_{0}}{\pi L_{\text {tomo }}}=25 \mathrm{~m}
$$

the associated eigenvalue diagram will include also eigenvalues with negligible energy, ensuring the presence of a noise subspace. Since the forested area presents an average tree height of $15 \mathrm{~m}$ and a maximum one of $22 \mathrm{~m}$ (i.e., less than $25 \mathrm{~m}$ ), subspace methods can be applied to perform tomography.

First, the Capon method has been applied for tomographic reconstruction [13]. All the tracks have been used for this purpose, and all the tomographic aperture length has been exploited. This nonparametric method is useful to obtain a reference image of the scene in order to validate the subsequent results obtained by means of the MUSIC algorithm. Referring to the tomogram (height-azimuth slice) of Fig. 10(a), one can observe that both ground and canopy contributions are clearly identifiable.

At this point, using the same amount of tracks, the MUSIC algorithm has been applied. Since only the maxima of the response have relevance, the dominant ones are shown in Fig. 10(b). The number of considered maxima corresponds to the number of eigenvalues having magnitude greater than ten percent of the maximum magnitude. The estimation is carried out adaptively for each snapshot. One can observe that the full aperture MUSIC response is consistent with the result obtained by means of the Capon method, and it allows one to determine the position and the extension of the two scattering components (ground, canopy). 


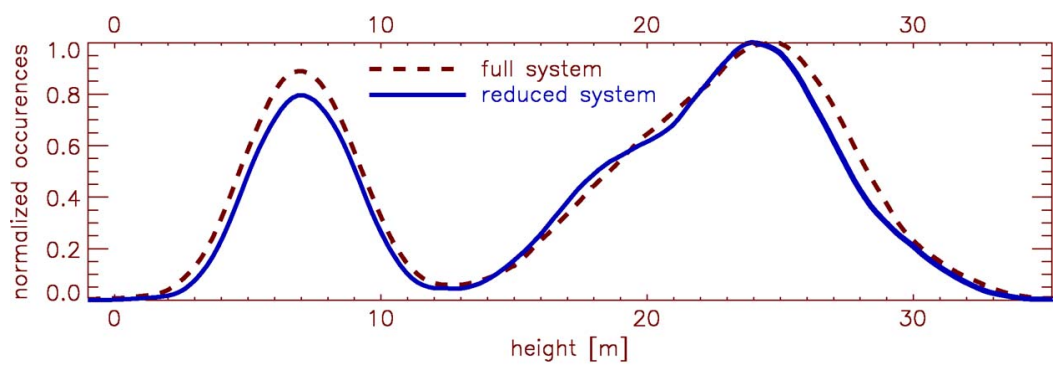

Fig. 11. Normalized average of the profiles along azimuth of (dashed line) Fig. 10(b) and (solid line) Fig. 10(c). The two distributed components related with ground and canopy have a Gaussian shape. This result is consistent with the assumed forest model and the estimated averaged tree height corresponds to the actual one (obtained by ground measurements).

For the reduced geometry $N=8$, the tomographic processing results are shown in Fig. 10(c). It is possible to observe that also with the reduced system, the two main contributions of the ground and the canopy are represented. Also, in this case, the dominant eigenvalues have been determined in order to perform the algorithm for each snapshot. For this case, it is interesting to observe that the averaged effective rank along the forested area is $R=4$, and the maximum one is $R=6$ [less than eight according to (28)].

Comparing Fig. 10(b) with Fig. 10(c), it is interesting to observe how the number of dominant components decreases proportionally with the tomographic aperture length as expected from (16). For this reason, the full system is capable to represent the forest layers with higher precision compared with the reduced one. In addition, the reduced aperture dimension increases the minimum angular separation of two close sources.

After the tomographic imaging is carried out, a validation of the chosen distributed source model can be done. Considering the full aperture MUSIC reconstruction described by the maxima and averaging them along the azimuth coordinate a representation of the presence of a scatterer for a determined height results (Fig. 11). It is possible to observe how, also with the reduced constellation, the two contributions can be clearly represented. In particular, a consistent estimation of their correspondent phase centers and widths is obtained. The presence of the two Gaussian distributions is in accordance with the model described in Section III and based on [17].

The length of the tomographic aperture has been determined in order to separate two contributions within a height of $6 \mathrm{~m}$ in the PLOS direction. Therefore, the detection of the position of two corner reflectors located with a PLOS height difference

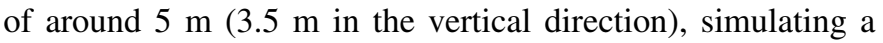
layover geometry (see Fig. 12), is carried out. Their response in the SAR image of Fig. 9 is represented by the bright spot inside the circle.

The results of the tomographic processing with reduced tracks are shown in Fig. 13. It is possible to observe that the relative height of the two corner reflectors can be correctly retrieved.

Since the tomographic aperture length is over dimensioned for this particular layover geometry, a further verification has shown that the two corner reflectors can still be separated with $N=5$ passes and a tomographic aperture of only $L_{\text {tomo }}=80 \mathrm{~m}$, as expected from (26).

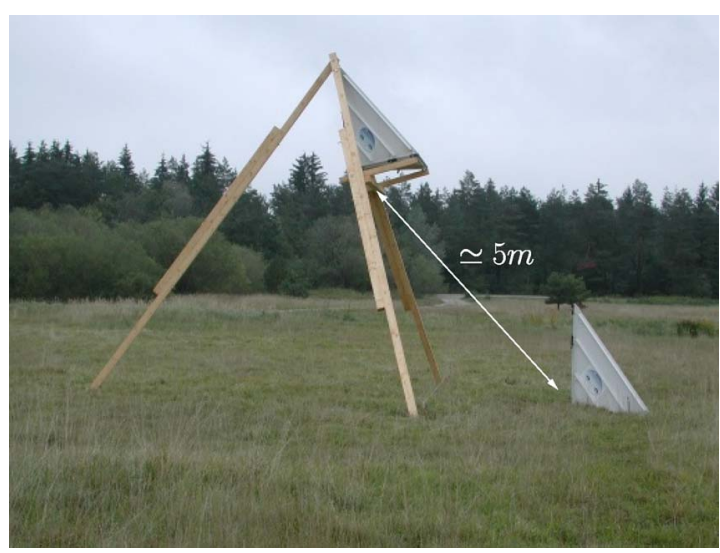

Fig. 12. Layover geometry. Two corner reflectors have been placed in a way that their response falls within the same range resolution cell. The vertical distance of the two phase centers is approximately $3.5 \mathrm{~m}$.

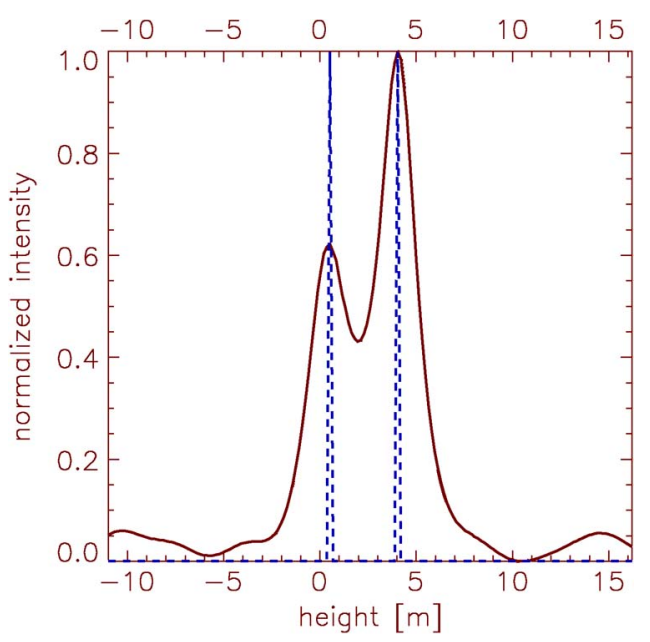

Fig. 13. Layover solution with the reduced tomographic aperture. (Solid line) MUSIC response and (dashed line) two maximum peaks.

\section{CONCLUSION}

In this paper, a detailed study on the dimension of a tomographic acquisition geometry has been carried out. The main parameters: minimum number of tracks, distance between the tracks and minimum tomographic aperture length have been determined. It has been shown that the identification of the effective signal and noise subspaces allows one to perform an 
analysis that leads to the estimation of the equivalent number of sources that the system has to solve when a distributed scatterer is viewed. Once the structure model has been defined, the analysis consists in the identification of the dominant components of the tomographic band limited signal; this task has been solved by means of the prolate spheroidal wave functions.

On the one hand, the knowledge of the minimum angular dispersion allows, by means of the related eigenvalues' behavior, to identify the minimum tomographic aperture length. On the other hand, the maximum angular dispersion identifies the minimum number of tracks and, consequently, the maximum baseline allowed to represent the smallest noise subspace.

The validation with the real data acquired by the E-SAR system of the DLR showed that, when subspace methods are applied, the proposed system reduction allows one to recover the same amount of information, as in the full acquisition case.

\section{APPENDIX A \\ GROUND AND CANOPY CONTRIBUTIONS}

The generic element of the covariance matrix [see (9)], under the independence assumptions can be written as

$$
\begin{aligned}
E\left[x(l) x^{*}\left(l^{\prime}\right)\right] & =\int_{-\frac{\pi}{2}}^{+\frac{\pi}{2}} \exp \left(j \frac{4 \pi\left(r_{l}(\phi)-r_{l^{\prime}}(\phi)\right)}{\lambda}\right) P(\phi) d \phi \\
& =\sum_{i=1}^{2} \alpha_{i} \int_{\theta_{i}+\Delta_{i}}^{\theta_{i}-\Delta_{i}} \exp \left(j \frac{4 \pi\left(l-l^{\prime}\right) \sin (\phi)}{\lambda}\right) \frac{1}{2 \Delta_{i}} d \phi \\
& =E\left[x_{\mathrm{c}}(l) x_{\mathrm{c}}^{*}\left(l^{\prime}\right)\right]+E\left[x_{\mathrm{g}}(l) x_{\mathrm{g}}^{*}\left(l^{\prime}\right)\right]
\end{aligned}
$$

where the amplitude difference between the two sources has been neglected. Applying the derivations of Section IV-A for the last two terms and noting that $L_{\text {tomo }}$ is the same for the two distributed scatterers, the value of $c$ is given from

$$
\begin{aligned}
c & =\frac{2 \pi \Delta_{\mathrm{c}} \cos \left(\theta_{1}\right)}{\lambda} L_{\text {tomo }}+\frac{2 \pi \Delta_{\mathrm{g}} \cos \left(\theta_{2}\right)}{\lambda} L_{\text {tomo }} \\
& \simeq \frac{2 \pi}{\lambda} L_{\text {tomo }}\left(\Delta_{\mathrm{c}}+\Delta_{\mathrm{g}}\right) \cos \left(\theta_{0}\right) \\
& =c_{1}+c_{2}
\end{aligned}
$$

where $\Delta_{\mathrm{c}}$ and $\Delta_{\mathrm{g}}$ are the angular dispersion of the canopy and the ground, respectively. Due to the assumed forest model, the greatest value of $c$ is directly proportional to $\Delta_{\mathrm{c}}+$ $\Delta_{\mathrm{g}}$ that is upper bounded by $\Delta$. Introducing the parameter $N_{\text {efs }}^{\text {tot }}$ as

$$
N_{\mathrm{efs}}^{\mathrm{tot}}=N_{\mathrm{efs}}^{\mathrm{c}}+N_{\mathrm{efs}}^{\mathrm{g}}=\left\lceil\frac{2\left(c_{1}+c_{2}\right)}{\pi}\right\rceil
$$

where $N_{\mathrm{efs}}^{\mathrm{c}}$ and $N_{\mathrm{efs}}^{\mathrm{g}}$ are the numbers of dominant eigenvalues contribution of the canopy and the ground, respectively, it is easy to see that the analysis carried out for a single uniformly distributed source produces, under the model assumptions, the widest spread of the signal energy among the dominant eigenvalues components. Therefore, it is sufficient to assume that the scene consists of a single uniformly distributed scatterer.

\section{APPENDIX B}

\section{SPACE-BANDWidTh PRODUCT DeRIVATION}

One of the most important points of this paper is related to the space-bandwidth product $c$. This appendix shows the derivation that led to (15). For the SARTom case, the eigenproblem to be solved is

$$
\begin{aligned}
& \int_{-\frac{L_{\text {tomo }}}{2}}^{+\frac{L_{\text {tomo }}}{2}} \exp \left(j \frac{4 \pi}{\lambda}\left(l-l^{\prime}\right) \sin \left(\theta_{0}\right)\right) \\
& \quad \cdot \frac{\sin \left(\frac{4 \pi}{\lambda}\left(l-l^{\prime}\right) \cos \left(\theta_{0}\right) \Delta\right)}{\frac{4 \pi}{\lambda}\left(l-l^{\prime}\right) \cos \left(\theta_{0}\right) \Delta} \Phi_{n}\left(l^{\prime}\right) d l^{\prime}=\mu_{n} \Phi_{n}(l) .
\end{aligned}
$$

Defining now

$$
\begin{aligned}
\Omega & =\frac{4 \pi \cos \left(\theta_{0}\right) \Delta}{\lambda} \\
\Phi_{n}(l) & =\exp \left(j \frac{4 \pi l}{\lambda} \sin \left(\theta_{0}\right)\right) S_{0 n}(l)
\end{aligned}
$$

where the functions $\Phi_{n}(l)$ are the modulated prolate spheroidal wave functions [19] and $S_{0 n}(l)$ are prolate spheroidal wave functions, it is possible to write (32) (neglecting some constant factors) as

$$
\int_{-\frac{L \text { tomo }}{2}}^{+\frac{L_{\text {tomo }}}{2}} \frac{\sin \left(\Omega\left(l-l^{\prime}\right)\right)}{\pi\left(l-l^{\prime}\right)} S_{0 n}\left(l^{\prime}\right) d l^{\prime}=\mu_{n} S_{0 n}(l)
$$

that is the expression analyzed in [22] where the spacebandwidth product is defined as

$$
c=\frac{\Omega L_{\text {tomo }}}{2} .
$$

Therefore, (15) results from (36) and (33).

\section{APPENDIX C \\ OVERLAP CASE}

If now, the hypothesis of no overlap between the different scatterers contributions is discarded, the expression of the main parameters will linearly change. It will be supposed that the angular dispersion of the ground component cannot be greater than the one of the canopy and that the phase center of the 


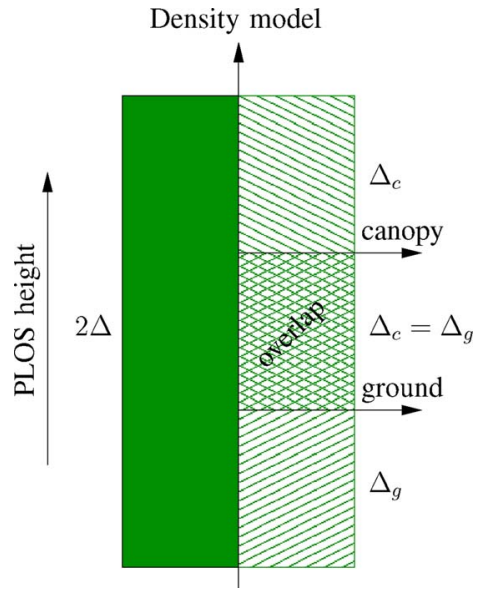

Fig. 14. Distributed scatterer model in case of source overlap.

ground cannot be inside the forest contribution (see Fig. 14). In formulas

$$
\left\{\begin{array}{l}
\Delta_{\mathrm{c}} \geq \Delta_{\mathrm{g}} \\
2 \Delta_{\mathrm{c}}+\Delta_{\mathrm{g}}=2 \Delta
\end{array}\right.
$$

the maximum overlap will result when $\Delta_{c}=\Delta_{g}$. Therefore

$$
\begin{aligned}
\Delta_{\mathrm{c}} & =\frac{2}{3} \Delta \\
\Delta_{\mathrm{c}}+\Delta_{\mathrm{g}} & =\frac{4}{3} \Delta .
\end{aligned}
$$

It is important to observe that $\sum_{i} \Delta_{i}$ will not change the volume sector $\Delta$ since the height of the volume remains the same, its impact is related to the assumptions on the scatterer structure. Therefore, the value of the maximum baseline will not change. Defining now

$$
\beta=\frac{\Delta_{\mathrm{c}}+\Delta_{\mathrm{g}}}{\Delta} \leq \frac{4}{3}
$$

we have that

$$
\begin{aligned}
N_{\text {efs }}^{\prime} & =\beta N_{\text {efs }} \\
L_{\text {tomo }}^{\prime} & =\beta L_{\text {tomo }} .
\end{aligned}
$$

The impact of this new model structure will be on $N_{\text {efs }}$ and consequently on $L_{\text {tomo }}$ since, as shown, the greater the tomographic aperture, the larger the number of viewable dominant eigenvalues.

At this point, since the baseline length did not change, it is necessary to proceed as in Section V and refer to (21) and (22) to determine the minimum system dimension. For the example reported in Section VI-A the number of required passes is $N=9$.

It is also possible to extend the overlap case to an arbitrarily number of sources that overlap for the half of their extension, in this case the parameter $\beta$ will be limited by $\beta \leq 2$.

It is worth to remember that the overlap case is more a theoretical generalization rather than a realistic one, because, due to the nature of the scatterers themselves that cannot occupy the same position within the 3-D volume, the model described in Section III is sufficient to describe the structure.

\section{ACKNOWLEDGMENT}

The authors would like to thank the DLR/E-SAR team and the anonymous reviewers for their constructive comments and suggestions.

\section{REFERENCES}

[1] A. Gabriel and R. M. Goldstein, "Crossed orbit interferometry: Theory and experimental results from SIR-B," Int. J. Remote Sens., vol. 9, no. 5, pp. 857-872, 1988.

[2] R. Bamler and P. Hartl, "Synthetic aperture radar interferometry," Inv. Prob., vol. 14, no. 4, pp. R1-R54, Aug. 1998.

[3] S. R. Cloude and K. P. Papathanassiou, "Polarimetric SAR interferometry," IEEE Trans. Geosci. Remote Sens., vol. 36, no. 5, pp. 1551-1565, Sep. 1998.

[4] A. Reigber and A. Moreira, "First demonstration of airborne SAR tomography using multibaseline l-band data," IEEE Trans. Geosci. Remote Sens., vol. 38, no. 5, pp. 2142-2152, Sep. 2000.

[5] A. Reigber, "Airborne polarimetric SAR tomography," Ph.D. dissertation, Univ. Stuttgart, Stuttgart, Germany, 2001.

[6] R. Horn, "The DLR airborne SAR project E-SAR," in Proc. IGARSS, Lincoln, NE, May 1996, vol. 3, pp. 1624-1628.

[7] G. Fornaro, F. Serafino, and F. Soldovieri, "Three-dimensional focusing with multipass SAR data," IEEE Trans. Geosci. Remote Sens., vol. 41, no. 3, pp. 507-517, Mar. 2003.

[8] G. Fornaro, F. Lombardini, and F. Serafino, "Three-dimensional multipass SAR focusing: Experiments with long-term spaceborne data," IEEE Trans. Geosci. Remote Sens., vol. 43, no. 4, pp. 702-714, Apr. 2005.

[9] F. Lombardini, "Differential tomography: A new framework for SAR interferometry," IEEE Trans. Geosci. Remote Sens., vol. 43, no. 1, pp. 3744, Jan. 2005

[10] F. Serafino, F. Soldovieri, F. Lombardini, and G. Fornaro, "Singular value decomposition applied to 4D SAR imaging," in Proc. IGARSS, Jul. 25-29, 2005, vol. 4, pp. 2701-2704.

[11] G. Fornaro et al., "Multidimensional imaging with ERS data," in Proc. FRINGE, Frascati, Italy, 2005.

[12] H. Krim and M. Viberg, "Two decades of array signal processing research: The parametric approach," IEEE Signal Process. Mag., vol. 13, no. 4, pp. 67-94, Jul. 1996.

[13] F. Lombardini and A. Reigber, "Adaptive spectral estimation for multibaseline SAR tomography with airborne L-band data," in Proc. IGARSS, Toulouse, France, 2003, pp. 2014-2016.

[14] S. Guillaso and A. Reigber, "Polarimetric SAR tomography," in Proc. POLINSAR, Frascati, Italy, Jan. 2005.

[15] A. Moreira, J. Mittermayer, and R. Scheiber, "Extended chirp scaling algorithm for air- and spaceborne SAR data processing in stripmap and ScanSAR imaging modes," IEEE Trans. Geosci. Remote Sens., vol. 34, no. 5, pp. 1123-1136, Sep. 1996.

[16] R. O. Schmidt, "Multiple emitter location and signal parameter estimation," IEEE Trans. Antennas Propag., vol. AP-34, no. 3, pp. 276-280, Mar. 1986.

[17] J. O. Hagberg, L. M. H. Ulander, and J. Askne, "Repeat-pass SAR interferometry over forested terrain," IEEE Trans. Geosci. Remote Sens., vol. 34, no. 2, pp. 331-340, Mar. 1995.

[18] Y. Meng et al., "Estimation of the directions of arrival of spatially dispersed signals in array processing," Proc. Inst. Elect. Eng.-Radar, Sonar Navig., vol. 143, no. 1, pp. 1-9, Feb. 1996.

[19] S. Valaee, B. Champagne, and P. Kabal, "Parametric localization of distributed sources," IEEE Trans. Signal Process., vol. 43, no. 9, pp. 2144 2153, Sep. 1995.

[20] T. P. Jantti, "The influence of extended sources on theoretical performance of the MUSIC and ESPRIT methods: Narrow band sources," in Proc. ICASSP, San Francisco, CA, Mar. 1992, vol. 2, pp. 429-432.

[21] M. Bertero and P. Boccacci, Introduction to Inverse Problem in Imaging. Bristol, U.K.: IOP Publ., 1998.

[22] D. Slepian and H. O. Pollak, "Prolate spheroidal wave functions, Fourier analysis and uncertainty-I," Bell Syst. Tech. J., vol. 40, pp. 43-64, Jan. 1961.

[23] D. Slepian and E. Sonnenblick, "Eigenvalues associated with prolate spheroidal wave functions of zero order," Bell Syst. Tech. J., vol. 44, pp. $1745-1759,1978$.

[24] M. Nannini et al., "Imaging of targets beneath foliage with SAR tomography," in Proc. EUSAR, Friedrichshafen, Germany, 2008.

[25] M. Nannini and R. Scheiber, "Height dependent motion compensation and coregistration for airborne SAR tomography," in Proc. IGARSS, Barcellona, Spain, 2007, pp. 5041-5044. 


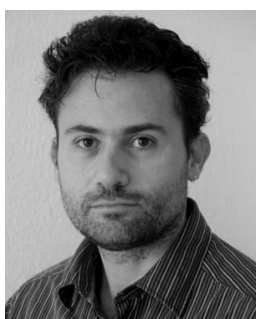

Matteo Nannini received the Laurea degree in telecommunication engineering from the University of Florence, Florence, Italy, in 2003, defending a thesis on the simulation of the receiver chain of a SAR system. The thesis has been done in collaboration with the Microwaves and Radar Institute (HR), German Aerospace Center (DLR), Oberpfaffenhofen, Germany.

Since 2004 he has been with the DLR-HR, where he is currently working on airborne synthetic aperture radar (SAR) processing and is pursuing the Ph.D. degree in collaboration with the University of Karlsruhe, Germany. His main research topics are SAR, SAR interferometry, and SAR tomography.

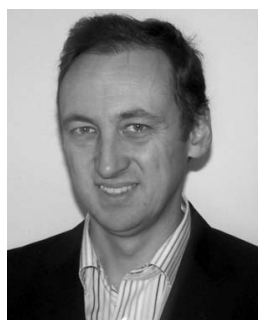

Rolf Scheiber received the Diploma degree in electrical engineering from the Technical University of Munich, Munich, Germany, in 1994 and the Ph.D. degree in electrical engineering from the University of Karlsruhe, Karlsruhe, Germany, in 2003, with a thesis on airborne synthetic aperture radar (SAR) interferometry.

Since 1994, he has been with the Microwaves and Radar Institute, German Aerospace Center, Oberpfaffenhofen, Germany, where he developed the operational high-precision interferometric SAR processor for its experimental SAR (E-SAR) airborne sensor. Since 2001, he has been heading the SAR signal processing group within the SAR Technology Department, where he is currently responsible for the E-SAR campaign data processing as well as the development of the processing software for the new airborne sensor F-SAR. His current research interests include algorithm development for high-resolution air- and spaceborne SAR focusing, SAR interferometry, differential SAR interferometry, SAR tomography, and radio sounding algorithms and applications.

Dr. Scheiber was awarded as coauthor with the 1996 Geoscience and Remote Sensing Society Transactions Prize Paper Award for the contribution "Extended Chirp Scaling Algorithm for Air- and Spaceborne SAR Data Processing in Stripmap and ScanSAR Imaging Modes” in 1997.

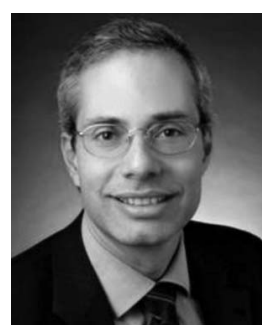

Alberto Moreira (M'92-SM'96-F'04) was born in São José dos Campos, Brazil, in 1962. He received the B.S.E.E. and the M.S.E.E. degrees from the Aeronautical Technological Institute, São José dos Campos, in 1984 and 1986, respectively, and the Eng. Dr. degree (with honors) from the Technical University of Munich, Munich, Germany, 1993.

In 2003, he received a full professorship from the University of Karlsruhe, Karlsruhe, Germany, in the field of microwave remote sensing. As its Chief Scientist and Engineer, he managed from 1996 to 2001 the Synthetic Aperture Radar (SAR) Technology Department,- Microwaves and Radar Institute, German Aerospace Center (DLR), Oberpfaffenhofen, Germany. Under his leadership, the DLR airborne SAR system, E-SAR, has been upgraded to operate in innovative imaging modes like polarimetric SAR interferometry and SAR tomography. Since 2001, he has been the Director of the Microwaves and Radar Institute, DLR. The Institute contributes to several scientific programs and space projects for actual and future air-and spaceborne SAR missions like TerraSAR-X and Sentinel-1. In 2006, the mission proposal TanDEM-X led by his institute has been approved for the realization phase. $\mathrm{He}$ is the Principal Investigator for this mission. His professional interests and research areas encompass radar end-to-end system design and analysis, innovative microwave techniques and system concepts, signal processing, and remote-sensing applications.

Dr. Moreira is serving as a member of the IEEE Geoscience and Remote Sensing Society (GRSS) Administrative Committee (1999-2001, 2004-2007, 2008-2009 as Executive Vice-President), was the Founder and Chair of the German Chapter of the GRSS (2003-2008), was an Associate Editor for the IEEE Geoscience AND Remote Sensing LetTers (2003-2007), and is serving as Associate Editor for the IEEE TRANSACTIONS ON GEOSCIENCE AND REMOTE SENSING. Since 2003, he has also been serving as a member of the Board of Directors of the Information Technology Society, German Association for Electrical, Electronic and Information Technologies. In 1995 , he was the recipient of the DLR Science Award. He and his colleagues received the GRSS Transactions Prize Paper Awards in 1997 and 2001, respectively. $\mathrm{He}$ is also the recipient of the IEEE Nathanson Award (1999) and the IEEE Kiyo Tomiyasu Award (2007). He has contributed to the successful series of the European SAR conferences since 1996 as a member of the Technical Program Committee, Technical Chairman (2000), Awards Chairman (2002-2004), General Chairman (2006), and Cochairman (2008). 